\title{
Fourier Transform Spectroscopy in the Visible Range
}

\author{
Jenny Magnes and Tyler Hatch \\ Physics and Astronomy Department, Vassar College, 124 Raymond Ave, Poughkeepsie, NY, 12538
}

\begin{abstract}
We present an undergraduate laboratory that utilizes Fourier spectroscopy with two lasers in the visible range to demonstrate the measurement technique. In addition to teaching an important spectroscopic tool, this laboratory exercise deepens the understanding of Fourier transforms. A Michelson interferometer is used to determine the 'unknown' wavelength of a green Helium Neon laser beam by scanning the position of a mirror and comparing the number of observed fringes with that from a known-wavelength red Helium Neon laser beam - the reference beam. The data points are recorded electronically using a photodiode and then transformed into frequency space where the unknown wavelength is calibrated against the known wavelength.
\end{abstract}

\section{INTRODUCTION}

Fourier Transform spectroscopy is typically performed in the InfraRed (FTIR) allowing for high-resolution broadband spectral analysis using weak signals. FTIR is especially useful when working with biological samples and polymers emitting in the InfraRed (IR). Spectral lines of biological samples in particular are very closely spaced and benefit from the high resolution of FTIR spectra. Researchers continue to use [1], perfect and expand [2] FTIR applications even though the technique took hold in the mid $20^{\text {th }}$ century $[3,4]$.

Here we present a Fourier Transform (FT) spectrometer in the visible range since it is easier to build, align and troubleshoot than an instrument in the IR range, which is not in the visible part of the spectrum. This FT spectrometer is ideal for combining the principles of FTs and Michelson interferometry in one experiment. The FT spectrometer collects all wavelengths simultaneously and is an alternative to dispersive spectrometers that consist of gratings or prisms.

The experimental challenge of co-propagating two optical beams through the Michelson interferometer combined with the conceptual and computational challenge of applying the FT to the data is ideal for an upper level undergraduate laboratory. The students learn about key optical components in the setup as well as alignment techniques such as 'walking the beam.' Calculating the frequency spectrum is a hands-on approach to relating to reciprocal space. The students determine the unknown wavelength by calibrating the spectral line(s) against the known frequency.

\section{THEORY}

The FT spectrometer is based on the principles of interference and FTs. Interference is applied in the Michelson interferometer, the experimental component, while the FT is used to transform the data into frequency space.

\section{A. Multiple Beam Interference}

In a conventional Michelson interferometer, a single laser beam is split into two beams. The beams are then recombined so that they form an interference pattern. The difference in Optical Path Lengths $(\triangle \mathrm{OPL})$ determines the interference pattern. A bright spot in the center indicates constructive interference where $\triangle \mathrm{OPL}$ is a multiple of the wavelength (Fig. 1). The interference pattern will shift by one fringe as $\triangle \mathrm{OPL}$ shifts by one wavelength. As $\triangle \mathrm{OPL}$ changes at a continuous rate the intensity will form a sinusoidal pattern over time at any point in the interference pattern.

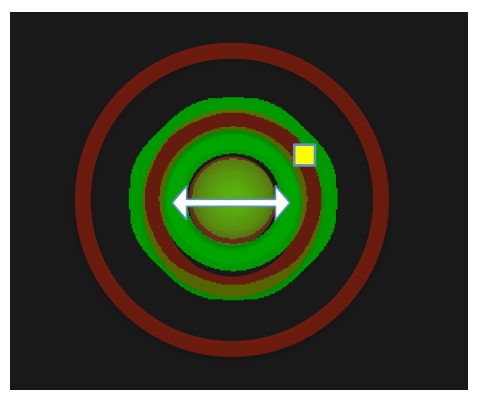

FIG 1. Model of an interferogram for two different wavelength co-propagating beams as observed in the FT spectrometer. The diameters of the circles would be increasing or decreasing as the $\triangle \mathrm{OPL}$ changes as emphasized by the double arrow. The square represents a point at which the intensity can be monitored as the interference rings change diameter.

In this FT spectrometer two beams of different wavelengths are sent into the Michelson interferometer. Each beam is split into two so that in the end four copropagating beams of two different wavelengths interfere. Monitoring the intensity at any part in the interference pattern creates two interfering sinusoids with different frequencies. The $\triangle \mathrm{OPL}$ occur at the same rate for all beams; however, the fringe shifts depend on the 
wavelengths. The fringes for each wavelength shift at different rates so that

$$
\Delta \mathrm{OPL}=m_{1} \lambda_{1}=m_{2} \lambda_{2}
$$

where $m_{1}$ and $m_{2}$ are the fractional shifts of the fringes while $\lambda_{1}$ and $\lambda_{2}$ are the respective wavelengths. The frequencies in the sinusoid observed by monitoring the intensity in the diffraction pattern are then in the same ratio as the wavelengths of the light used to create the interference pattern.

\section{B. Fourier Analysis}

Any function, $F(t)$, that obeys the Dirichlet conditions [4, 5] can be represented by a series of sine and cosine functions, which is known as the Fourier series [5, 6].

$$
F(t)=\sum_{n=-\infty}^{\infty} a_{n} \cos \left(2 \pi n v_{0} t\right)+b_{n} \sin \left(2 \pi n v_{0} t\right),
$$

where $v_{0}$ is the fundamental frequency, $a_{n}$ and $b_{n}$ are the amplitudes associated with each sine and cosine, $t$ is the variable representing time and $n$ an integer number. This summation can be written in a more succinct form using Euler's formula:

$$
F(t)=\sum_{m=-\infty}^{\infty} C_{m} \mathrm{e}^{2 \pi i m v_{0} t}
$$

where $C_{m}$ are complex coefficients and $\mathrm{m}$ is the integer used to sum from negative to positive infinity. The series of coefficients $C_{m}$ associated with each frequency is known as the FT.

In the case of digital data acquisition with a finite number of points, $N$, the infinite series of coefficients $C_{m}$ can be approximated with a finite series, $\boldsymbol{X}$, using a discrete FT (DFT) [6, 7]:

$$
X_{k}=\sum_{n=0}^{N} x_{n} \mathrm{e}^{-\frac{2 \pi i k n}{N}}
$$

where $X_{k}$ is the $k^{\text {th }}$ element in Fourier space, $x_{n}$ is the $n^{\text {th }}$ element in the object space and $N$ represents the total number of elements. The Fast Fourier Transform (FFT) takes advantage of the symmetrical characteristics of the FT to speed up the computation of a DFT.

The FT identifies the frequencies contained in the sine and cosine functions that the Fourier series describe. In this experiment, the intensity profile at any point in the interferogram consists of only two superimposed sinusoidal functions; hence, the FT of that intensity profile consists of just two frequencies as shown in Fig. 2.
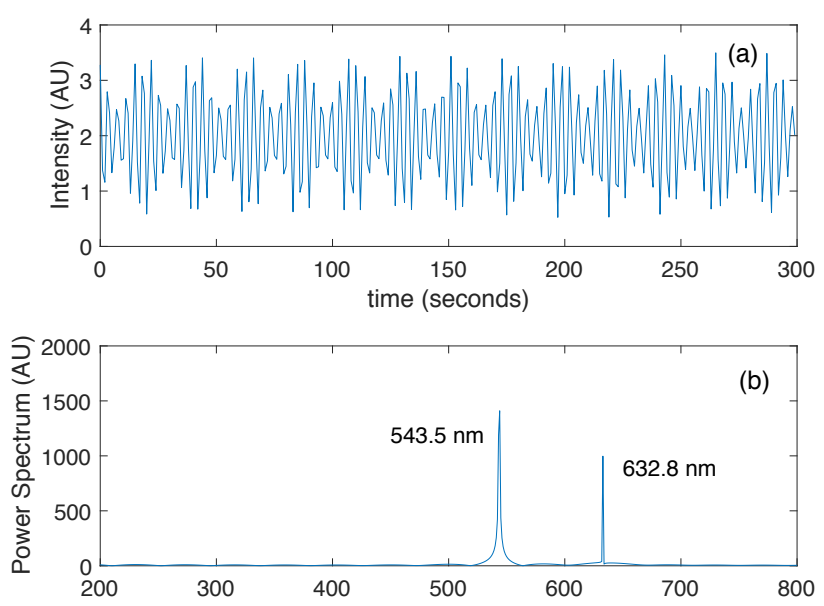

FIG 2. (a) Two superimposed sinusoids. (b) The FT of the sinusoids where the red laser is used to scale the horizontal axis.

\section{FT SPECTROMETER}

The experimental setup is customized for the use of two Helium-Neon (HeNe) lasers $(632.8 \mathrm{~nm}$ and $543.5 \mathrm{~nm}$ by Melles Griot). One laser is of 'unknown' frequency and the calibration laser has a known frequency. Both laser beams are combined using a beamsplitter and sent into the Michelson interferometer as shown in the experimental setup in Fig. 3.

Each beam is split into two beams using a second beamsplitter. The beams travel along each of the two arms of the Michelson interferometer and are turned around with front surface mirrors to be re-combined at the same beamsplitter that was used to split the beams. The optical alignment is the biggest experimental challenge in this experiment. The students have to learn the importance of maintaining an optical plane as well as walking the beams to center them on the mirrors and beamsplitters. 


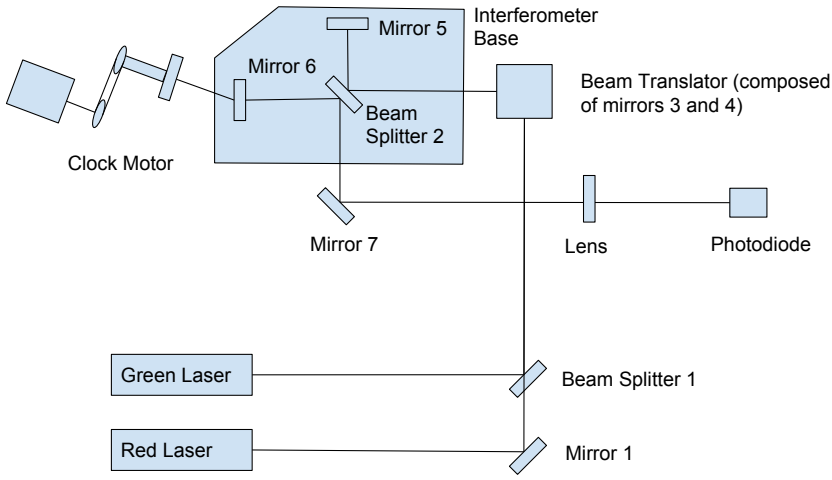

FIG 3. Experimental setup of FT spectrometer: Two different color laser beams co-propagate through the Michelson interferometer.

The combined beams are then expanded using a lens so that a photodiode (PD) can be placed in the interference pattern (Fig. 1). One of the mirrors in the interferometer arms is attached to a clock motor that allows for movement along the arm changing the $\Delta O P L$. LabView collects the data via the PD as the rings are changing diameters.
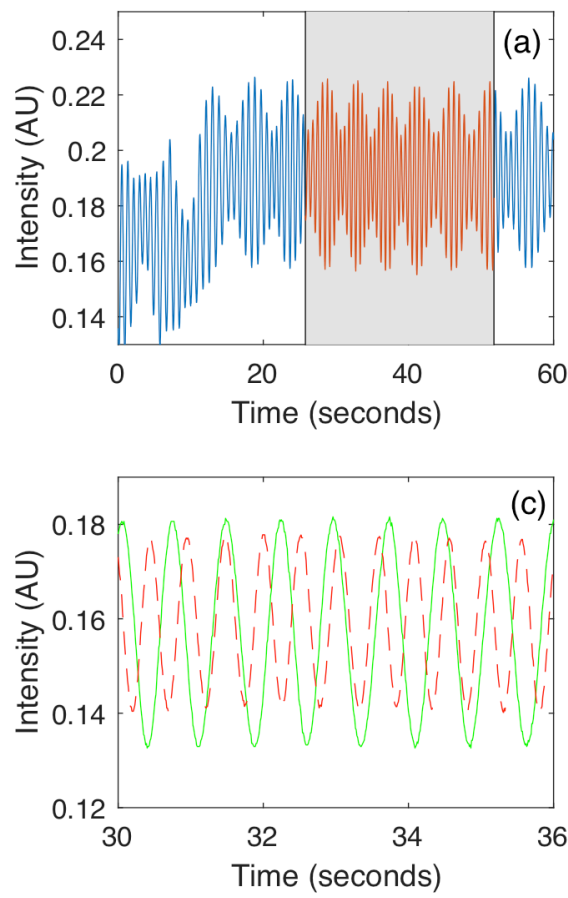

\section{DATA ANALYSIS AND DISCUSSION}

Monitoring the intensity of the combined beams yields a superposition of two sinusoids. The beat frequency is visible in Fig. 4(a) similar to the simulated data (Fig. 2(a)). The irregular data in the first 15 seconds (Fig. 4(a)) indicates that the clock motor skips and/or slips on occasion. This is an opportunity for the students to consider experimental factors that are usually unaccounted for in the theoretical models. Calculating the FFT of the full data set without screening renders multiple shifted peaks since the speed of the clock motor is inconsistent (Fig. 4(b)). Selecting a data segment that is consistent (shaded segment) in Fig. 4(a) results in two distinguishable peaks in the FT spectrum (solid line in Fig. 4(b)).

The uncalibrated FT spectrum for the selected stretch of data yielded a peak at $1.655 \pm 0.0195 \mathrm{~Hz}$ for the known $\mathrm{HeNe}$ laser at $632.8 \mathrm{~nm}$, which is the calibration laser. The peak of the 'unknown' wavelength was found at $1.424 \pm$ $0.0195 \mathrm{~Hz}$ so that the measured wavelength of the 'unknown' HeNe laser is $544.5 \pm 19.5 \mathrm{~nm}$. The measured value falls well within the error margins. The error associated with the entire data set is $0.009 \mathrm{~Hz}$, which is 8.5 $\mathrm{nm}$ for the green wavelength; however, the systematic error introduced by the slipping clock motor outweighs the uncertainty introduced by the length of the data set. In a
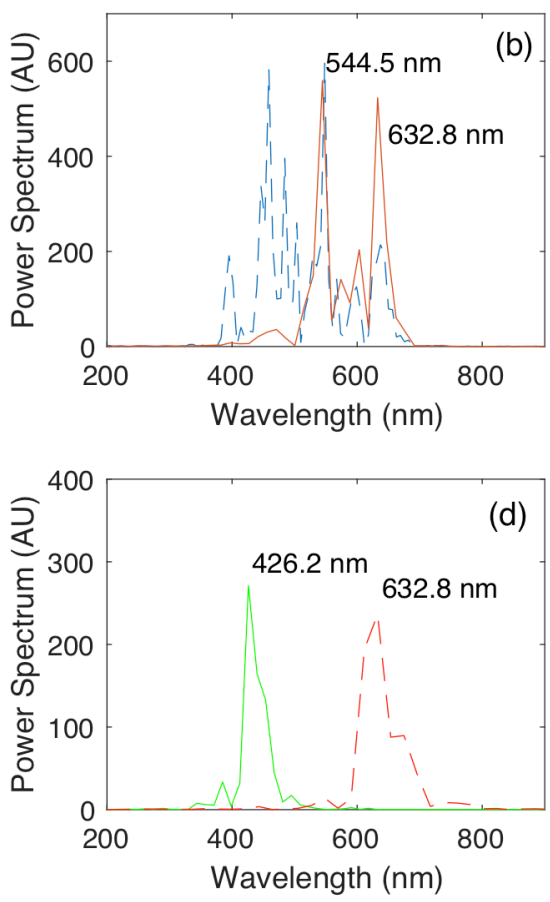

FIG 4. (a) The raw data of two interfering waves exiting the FT spectrometer. (b) The calibrated FT (solid line) indicates the FT of the shaded data. (c) Data taken consecutively at $632.8 \mathrm{~nm}$ (dashed line) and $543.5 \mathrm{~nm}$ (solid line). (d) Superimposed FT spectra of consecutively scanned laser lines. One laser is blocked while the other is recorded instead of recording the laser lines simultaneously. 
commercial FTIR the motors are very precise, and the scanning distance can be several meters to ensure high frequency resolution.

Figure 4(c) shows the time dependent laser profile recorded during different trials instead of simultaneously as in Fig. 4(a). The independent intensities show no beat frequency. The red laser oscillates at a higher frequency than the green laser as can be seen in Fig. 4(c). The frequencies are not synchronized in the independent run since the two lasers are not scanned simultaneously so that the calibration in Fig. 4(d) is not as accurate as in Fig. 4 (b).

The resolution of the FT depends on the extent of the data set. The range of the entire data set is 60 seconds so that the uncertainty in the frequency of the FT is $1 / 60$ (0.017) $\mathrm{Hz}$ while the selected data set only covers 25.98 seconds, resulting in an uncertainty of $0.038 \mathrm{~Hz}$. The rate at which the data points are collected must satisfy the Nyquist condition $[7,8]$, which states that the sampling frequency must be more than twice the signal frequency.

\section{CONCLUSIONS}

This advanced undergraduate laboratory has been taught for well over a decade at Vassar College. The students complete a Michelson interferometry laboratory before engaging in this exercise during which they learn about optical alignment and the physics behind the setup. The students have a very vague understanding of FTs prior to moving on to the FT spectrometer.

This experiment helps the students refine their optical alignment skills since two different wavelength laser beams must be aligned in the interferometer. The students learn to appreciate the FT as a useful tool. Achieving a frequency resolution of several nanometers is impressive with relatively inexpensive equipment. Another highly valuable component during this exercise is the combination of experimental errors. The fact that a longer scanning distance does not necessarily render better results is often baffling to the students.

Overall, this laboratory exercise covers all the essential components while inexpensive. A number of the components can be replaced with even more accessible components such as diode lasers and programmable hardware.

\section{ACKNOWLEDGEMENTS}

We are grateful for the support of the Vassar College Undergraduate Research Summer Institute (URSI), the Lucy Maynard Salmon Research Fund. We thank Mary Jikhars for editorial support.

[5] J.F. James, A Student's Guide to Fourier Transforms with Applications in Physics and Engineering, Cambridge University Press, Cambridge (1995).

[6] E.O. Brigham, The fast Fourier transform, PrenticeHall, Englewood Cliffs, NJ, (1974).

[7] R.H.T. Bates, Optik 67, 3, 247 (1982).

[8] J. Miao, D. Sayre, and H.N. Chapman, J. Opt. Soc. Am. A 15, 6, 1662 (1998).

[3] J.L. Koenig, Appl. Spectr 29, 4, pp. 293-309 (1975).

[4] A.V. Oppenheim, A.S. Willsky and Syed Hamish Nawab, Signals \& Systems, Prentice Hall. p. 198 (1997). ISBN 9780136511755 